\title{
Abstracts Presented at the International Neuropsychological Society, British Neuropsychological Society and the Division of Neuropsychology of the British Psychological Society Joint Mid-Year Meeting
}

\author{
July 6-9, 2005 \\ Dublin, Ireland
}

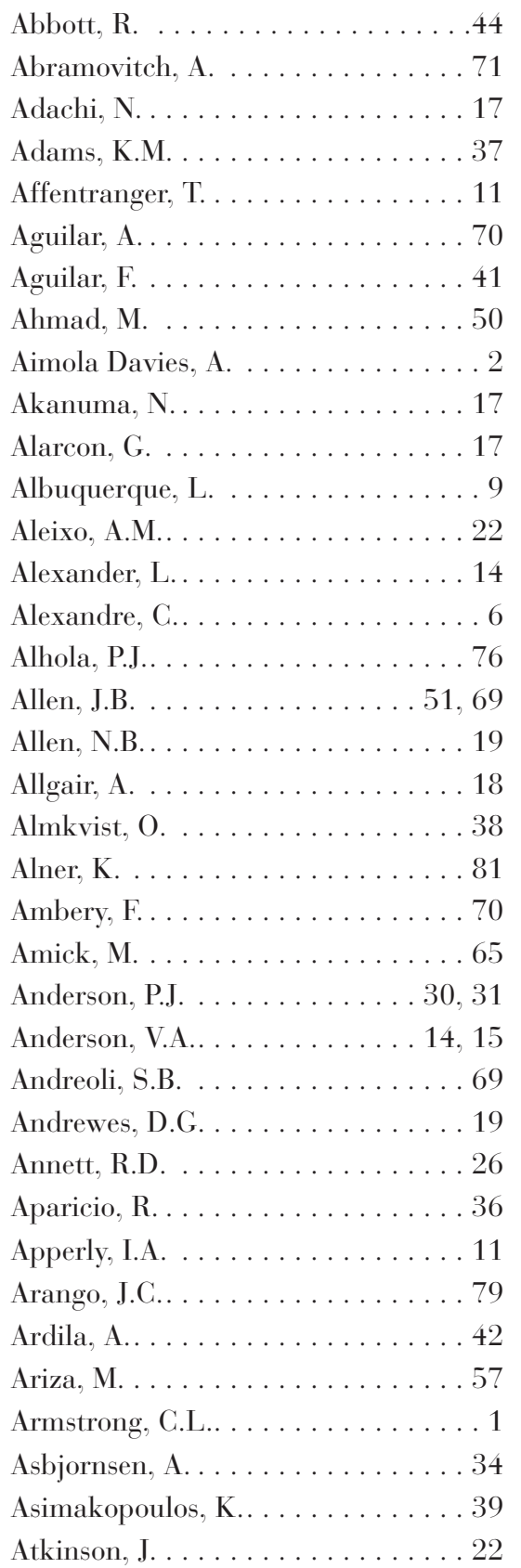

Austin. N.C.. . . . . . . . . . . . 31

Autti-Ramo, I. . . . . . . . . . . 30

Avanzini, G.............. . . 50

Baddeley, A.D. . . . . . . . . 81

Badwan, D. . . . . . . . 48, 49

Bakker, K. . . . . . . . . . . 27

Balconi, M. . . . . . . . 19, 23

Bangert, B. . . . . . . . . . . 28

Banks, S. . . . . . . . . . 34

Bannister, C. . . . . . . . . . . 27

Bargallo, N. . . . . . . . . 28, 57

Barker, L.A. . . . . . . . . . . . . . 52

Barnes, M. . . . . . . . . . . . . . 44, 45

Barrett, A.M. . . . . . . . . . 5, 64

Bartres-Faz, D. . . . . . . . . 2

Bastin, C.............. 8

Bateman, A. . . . . . . . . . 77

Baum, C. . . . . . . . . . . . 59, 60

Bayless, J.D. . . . . . . . . . . . . 2

Baynes, K. . . . . . . . . . . . 21

Beadle, G. . . . . . . . . . . . . . . 39

Bear, M. . . . . . . . . . . . 31

Bechara, A. . . . . . . . . . . . . 20

Beebe, D.W. . . . . . . . . . . . . . 29

Beeckmans, K. . . . . . . 55, 58

Beglinger, L.J. . . . . . . . . . . . . 2

Bell, T. . . . . . . . . . . . . 33

Bellamy, N. . . . . . . . . . . . 14

Bender, B.G. . . . . . . . . . . . 26

Bennett, H.E.. . . . . . . . . . . . . 59

Berninger, V. . . . . . . . . . . 44

Berninger, V.W. . . . . . . . . . 43

Bertolucci, P.Н. . . . . . . . . . . . 69

Beyens, Н. . . . . . . . . . . . 58

Bianchi, A. . . . . . . . . . . 27

Bieliauskas, L.A. . . . . . . . . 70
Biessels. G. . . . . . . . . . . 38, 39

Bigler, E.D. . . . . . . . . . . . . 18

Black, S.E. . . . . . . . . . . 52, 53

Blackman, J.A. . . . . . . . . . . . . 29

Blanco-Menendez, R. . . . . . . . . . 12

Boeka, A. . . . . . . . . . . . 14

Bolewska, A. . . . . . . . . . . 77

Boltes, A. . . . . . . . . . . . 61

Bonvallat, J.. . . . . . . . . . . . 17

Borden, K.A. . . . . . . . . . . . . 29

Bornstein, R. . . . . . . . . . . . . 42

Botet, F. . . . . . . . . . 7, 28

Bottoms, J.. . . . . . . . . . . . . . . 51

Bottoms, J.M. . . . . . . . . . . . . 51

Bouffet, E. . . . . . . . . . . . . . 30

Bouma, A. . . . . . . . . . . . . . 39

Bourne, V.J. . . . . . . . . . . . 74

Bowen, A. ............8

Bowie, G. . . . . . . . . . . 32

Boyd, J. . . . . . . . . . . . . . 2

Bradshaw, C. . . . . . . . . . . 34

Bradshaw, J.L. . . . . . . . . . . . 65

Braga, L.W. . . . . . . . . . . . 18

Bramao, M. . . . . . . . . 6, 10

Branch, A. . . . . . . . . . . 14

Brands, I. . . . . . . . . . . . . 39

Braybrooke, S. . . . . . . . . 64

Brenda, M. . . . . . . . . . . . . 68

Bressand, V. . . . . . . . . . . 20

Briggs, R. . . . . . . . . . . . 21

Brockhaus, R. . . . . . . . . . 69

Brockington, J.C. . . . . . . . . . . 60

Brouwer, W. . . . . . . . . . . 4, 12

Brouwer, W.H. . . . . . . . . . . 71

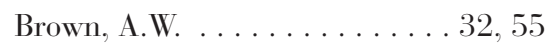

Brown, R. . . . . . . . . . 75

Brown, W.H. . . . . . . . . 12 
Bruce, I. . . . . . . . . . . . . . . . . 62

Brugger, P. . . . . . . . . 3, 4, 19

Buck, M.L. . . . . . . . . . . . . . 29

Bull, R. . . . . . . . . . . . . . 45

Bultitude, J.H. . . . . . . . . . . . 2

Burgers, I. . . . . . . . . . . . . 75

Burgess, P.W. . . . . . . . 46, 66, 67

Burrack, A.K. . . . . . . . . . . 3, 19

Burton, . . . . . . . . . . . . 20

Burton, M. . . . . . . . . . . . . 20

Busquets, N. . . . . . . . . . . 58

Butler, R.W. . . . . . . . . . . . . . . . 29

Cahill, L. . . . . . . . . . . 58

Caine, D. . . . . . . . . . . . . 3

Caldu, X............... 2

Carroll, E. . . . . . . . . . . . . . 80

Carstairs, J.R. . . . . . . . . . . 69

Carton, S. . . . . . . . . 7, 58

Cartwright-Hatton, S. . . . . . . . . 72

Catroppa, C. . . . . . . . . . 15

Caveney, A.F. . . . . . . . . . . . 70

Chan, R.C.. . . 19, 36, 45, 46, 57, 69

Charak, D.A. . . . . . . . . 28, 32

Charlton, J.L. . . . . . . . . . 65

Charman, T. . . . . . . . . . . . 46

Charnallet, A. . . . . . . . . . 8

Chater, N. . . . . . . . . . . . . 45

Chatterjee, A. . . . . . . . 24, 25

Che Din, N. . . . . . . . . . . . . 50

Chelune, G. . . . . . . . . . . . 81

Chelune, G.J. . . . . . . . . . . . . . 52

Chen, E.Y. . . . . . . . . . 19, 36

Chey, J. . . . . . . . . . . . . . . . . . 60

Chiaravalloti, N. . . . . . . . . . . 79

Chin. A. ............... 62

Choudhry, R.K. . . . . . . . . . 13

Christensen, B. . . . . . . . 67,68

Chu, Z.X. . . . . . . . . . . 18

Chung, J. . . . . . . . . . . . .8

Ciampitti, M. . . . . . . . . . . . 22

Cipolotti, L. . . . . . . . . . . 81

Cirino, P. . . . . . . . . . . . . 44

Clare, L. . . . . . . . . . . . . . . 54, 80

Clarkson, A.J. . . . . . . . . . . . . 50

Clemente, I. . . . . . . . . . . . . . . 2

Cluydts, R. . . . . . . . . . . . 55

Coakley, D. . . . . . . . . . . 62

Cockburn, J. . . . . . . . . . . . 2

Coen, R.F. . . . . . . . . . . . 62

Cole, S. . . . . . . . . . . . . . 10
Collette, F. . . . . . . . . . . 63

Collins, M. . . . . . . . . . . . 40

Colombo-Thuillard, F. . . . . . . . 78

Coltheart, M. . . . . . . . . . . . 24

Conaway, M.R. . . . . . . . . . . 29

Connor, L.T. . . . . . . . . . . . . . 59

Conway, M.A. . . . . . . . . . 16

Cook, M. . . . . . . . . . . . 58

Coolican, J. . . . . . . . . . . . . 5

Cooper, T.J. . . . . . . . . . . . .6 6

Corballis, M.C. . . . . . . . . 4

Corballis, P.M. . . . . . . . . . . . 4

Corker, T. . . . . . . . . . . 81

Corney, P. . . . . . . . . . 75

Cory, E. . . . . . . . . . . . . 64

Coslett, H. . . . . . . . . . 24, 25

Counet, N. . . . . . . . . . . . . . 44

Couper, E. . . . . . . . . . . 15

Cragar, D.E. . . . . . . . . . . 71

Crawford, J. . . . . . . . . . . . 69

Crawford, J.R. . . . . . . . 24, 32, 81

Croker, V. . . . . . . . . . . 16

Crossley, M. . . . . . . . 3, 4, 75

Crosson, B. . . . . . . . . . . 21

Crucian, G.P. . . . . . . . . . . . 64

Cumming, S. . . . . . . . . . 34

Cunningham, C. . . . . . . . . 62

Currie, D. . . . . . . . . . . . 24

D'Souza, S. . . . . . . . . . 27

D'Ydewalle, G. . . . . . . . . 66, 67

Daniels, B.J.. . . . . . . . . . . . 21

Darby, F. . . . . . . . . . . 10

David, S. . . . . . . . . . . 81

Davis, C. . . . . . . . . . . . 21

Davis, K. . . . . . . . . . . . 26

Dawes, S.............. . 81

De Bruycker, W. . . . . . . . . 67

De Goede, M. . . . . . . . . . . . . 12

De Haan, E.F. . . . . . . . . . . . . . 38

De Haan, E.H. . . . . . . . . 22, 24, 39

De la Fuente, I. . . . . . . . . . . . 58

De Lamotte, M. . . . . . . . . . . . . . 63

Dean, T. . . . . . . . . . . . 33

Deary, I.J. . . . . . . . . . . . . 74

Deijen, J. . . . . . . . . . . . 75

Dekker, R. . . . . . . . . . . 4

Delevoye-Turrell, Y. . . . . . . . . 37

DeLuca, J. . . . . . . . . . . 51, 79

Den Hollander, J.A. . . . . . . . . . 60

Denby, C................ 8
Deng, V.Y. . . . . . . . 4 45, 57, 69

Dennis, M. . . . . . . . . . 45, 73

Dent, C................. 29

Desbois, J. . . . . . . . . . . . . 55

Desmarais, G. . . . . . . . . 52,53

Devriese, P. . . . . . . . . . . . 75

Dewar, B.............. . 70

Dickson, A. . . . . . . . . . 27

Dietrich, A. . . . . . . . . . . 28

Dietrich, K.N. . . . . . . . . . 40

Diggle, P. . . . . . . . . . . . 35

Dijkerman, C. . . . . . . . . . . 53

Dikmen, S.S. . . . . . . . . . . . 56

Ding, Y.L. . . . . . . . . . . . 19

Dixon, M.J. . . . . . . . . . 52, 53

Dob, R. . . . . . . . . . . 14

Dockree, P. ............ .

Doniger, G.M. . . . . . . . . . . . 71

Donnelly, J. . . . . . . . . . . . 64

Donnelly, K............... 64

Dooley, J. . . . . . . . . . . . . 14, 15

Dorris, L. . . . . . . . . . . . 27, 28

Downey, R.A.............. . 21

Doyle, L.W. . . . . . . . . . . . . . . 31

Dromerick, A. . . . . . . . . . . 59

Drotar, D. . . . . . . . . . . . 15

Drotar, N.D.. . . . . . . . . . . . . 32

Druks, J. . . . . . . . . . . . 80

Duff, K. . . . . . . . . . . . 2

Dumontheil, I. . . . . . . . . . 67

Duquette, J. . . . . . . . . . . . 71

Duquin, J. . . . . . . . . . . 64

Dyer, K.R. . . . . . . . . . 40

Edgin, J. . . . . . . . . . . . 30

Edwards, D.F. . . . . . . . . 59, 60

Edwards, M.G............... . 3

Egeland, J.. . . . . . . . . . . . . . 34

Egleston, P.N. . . . . . . . . . . 12

Ek, L. . . . . . . . . . . . 38

Elgin, J. . . . . . . . . . . . . . 31

Eliez, S. . . . . . . . . . 20

Elkadi, S. . . . . . . . . . . 40

Ellefson, M. . . . . . . . . . . 45

Ellis, J.A. . . . . . . . . . . . . 66

Emslie, H. . . . . . . . . . . 56

Eranti, S................ 35

Erasmus, A.............. 33

Eren, S. . . . . . . . . . . 14

Erhan, H.M. . . . . . . . . . . 65

Ervalahti, N. . . . . . . . . . . 30 
Escamilla-Sevilla, F. . . . . . 65, 66

Eskes, G.A. . . . . . . . . . . 5

Espy, K. . . . . . . . . . . 32

Espy, K.A. . . . . . . . . 28, 30, 45

Estevez-Gonzalez, A. . . . . . . . . 61

Evanochko, W.T. . . . . . . . . . . 60

Evans, J. . . . . . . . . . . . . . . . 46

Evans, J.J. . . . . . . . . . . . 25, 33

Ewing-Cobbs, L. . . . . . . . . . . . . 44

Eynon, S. . . . . . . . . . . . . . 79

Fabri, M. . . . . . . . . . . 4

Fagerlund, A.M. . . . . . . . . . 30

Faisca, L.M. . . . . . . . . 6, 10

Farias, D. . . . . . . . . . 21

Feijen, J. . . . . . . . . . . 61

Fernandes, J. . . . . . . . . . . . . . 32

Fields, J.A. . . . . . . . . . . . 64

Firminger, S. . . . . . . . . . 26

Fish, J. . . . . . . . . . . . . . 56

Flaherty-Craig, C. . . . . . . . . . 5

Flaherty-Craig, C.V. . . . . . . . . . 52

Fleming, J. . . . . . . . . . . . . 55

Fletcher, J.M. . . . . . . . . 44, 45, 73

Foley, J.A. . . . . . . . . . . . 43

Forbes, C. . . . . . . . . 67

Forman, A.C............. 56

Foster, J.K. . . . . . . . . . . 8

Fotopoulou, A........... 16

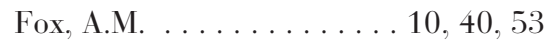

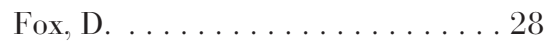

Fox, H.C.. . . . . . . . . . . 74

Frasca, A. . . . . . . . . . 42

Frasca, D. . . . . . . . . . . . 68

Freeland, J.C. . . . . . . . . . . . 81

Freeman, J.E. . . . . . . . . . . 66

Friedman, T.W. . . . . . . . . 62

Frijns, C.J. . . . . . . . . . . 16

Fuchs, L. . . . . . . . . . . 44

Fujii, T. . . . . . . . . . 5, 10

Fulwiler, C. . . . . . . . . . . . . 18

Funnell, E. . . . . . . . . 80, 81

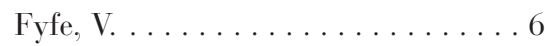

Gade, A. . . . . . . . 47, 62, 63

Gaiefsky, M.............. . 21

Gallagher, P. . . . . . . . . . . . 35

Gansler, D... . . . . . . . . . . . 18

Gao, D.G. . . . . . . . . . . . . 19

Garavan, H. . . . . . . . . 4 46,58

Garcia-Sanchez, C. . . . . . . . 61
Garley, C. . . . . . . . . . . 81

Garrard, P.. . . . . . . . . . . 80

Gaudino-Goering, E. . . . . . . . . 51

Geffen, G. . . . . . . . . . . . 73

Gelinas, I. . . . . . . . . . . . 71

Genetti, M. ............. 78

Georgiev, S. . . . . . . . . . . 41

Gibbie, T. . . . . . . . . . . 43

Gibson, M. . . . . . . . . . . . . . . 72

Giersch, A. . . . . . . . . . . 37

Gilbert, S.J. . . . . . . . . . . 67

Giles, S. . . . . . . . . . . . 5

Gill-Thwaites, H. . . . . . . . 48, 49

Gillespie, D.C. . . . . . . . . . . 8

Gimenez, M. . . . . . . . . . . . 28

Giordani, B... . . . . . . . . . 70

Giovagnoli, A. . . . . . . . . . . . 50

Giovagnoli, A.R. . . . . . . . . . . 13

Goldstein, L.H. . . . . . . . . . . 48, 70

Gomez Rio, M. . . . . . . . . . . . . 37

Gomez-Ruiz, I. . . . . . . . . . . 70

Gonzalez Rothi, L.J. . . . . . . . . . 22

Gonzalez-Rothi, L.J. . . . . . . . . . . 21

Goodkin, K. . . . . . . . . . . . 42

Gopinath, K. . . . . . . . . . 21

Gordon, M. . . . . . . . . . . 26

Gorny, I. . . . . . . . . . . . . . 69

Goverover, Y. . . . . . . . . . . 51, 79

Grace, J. . . . . . . . . . . . . 65

Gracey, F.. . . . . . . . . . 33, 77

Grayson, S. . . . . . . . . . . 17

Green, R. . . . . . . . . 67,68

Green, R.D. . . . . . . . . . . . 12

Greene, E. . . . . . . . . . . . 62

Griffith, R. . . . . . . . . . . 60

Guo, M. . . . . . . . . . . . 45, 57

Guo, M.Y. . . . . . . . . . . . . 69

Gurka, M.J. . . . . . . . . . . . . . . 29

Gustafson, L. . . . . . . . . . . . 17

Gutwin, C.............. 4

Gydesen, S. . . . . . . . . . 47

Haber, A.H. . . . . . . . . . . . . . 13

Hachey, N. . . . . . . . . . . . . 33

Hack, M. . . . . . . . . . . . . . . 32

Hahn, M. . . . . . . . . . . . . . 60

Hallett, W. . . . . . . . . . . . 17

Hamilton, J.M. . . . . . . . . . . . . 35

Hammond, G. . . . . . . . . . . 53

Hanks, R.A. . . . . . . . . . . . 13

Hanna-Pladdy, B. . . . . . . . . . 53
Hannesdottir, K. . . . . . . . . . 54

Hanten, G. . . . . . . . . . . . . 18

Harciarek, M. . . . . . . . . . . 23

Haritou, F. . . . . . . . . . 15

Harley, C. . . . . . . . . . . . . 2

Harrell, L.E. . . . . . . . . . . . . 60

Harrington, G.S. . . . . . . . . 21

Harris, A. . . . . . . . . . . . . 72

Harris, L. . . . . . . . . . . . . 34

Hart, S. . . . . . . . . . . . . . 80

Harve-Rytsola, H. . . . . . . . . . . 40

Harvey, M. . . . . . . . . . . . . . 6

Harvey, S. . . . . . . . . . . . . 15

Hashimoto, R. . . . . . . . . . 10

Hatta, T. . . . . . . . . . 9, 76

Hawkins, K. . . . . . . . . . 46

Heald, G.R. . . . . . . . . . . . . 1

Heilman, K.M. . . . 22, 24, 25, 53, 64

Hejl, A. . . . . . . . . . . . 63

Hennerici, M.G. . . . . . . . . . . 71

Henry, J.C.. . . . . . . . . . . . . 70

Henry, M. . . . . . . . . . . . . . . 69

Henselmans, J.M. . . . . . . . . . . . 39

Henwood, K. . . . . . . . . . . . . 33

Hepp-Reymond, M. . . . . . . . . . 3

Herbert, C.M. . . . . . . . . . . . 49

Hernandez, M. . . . . . . . . . . . 62

Hess, A.L. . . . . . . . . . . . . 38

Hillary, F.G. . . . . . . . . . . . . 79

Hirayama, K. . . . . . . . . . . . 10

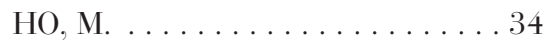

Hodges, J.R. . . . . . . . . . . 80

Hof, A. . . . . . . . . . . 4 4

Holland, L. . . . . . . . . . . . . . . 32

Hoogma, R.P. . . . . . . . . . . . . 39

Hopkins, R.O. . . . . . . . . . . . 18

Howard, K. . . . . . . . . . . . . 31

Hoyme, E. . . . . . . . . . . . . 30

Hugdahl, K.. . . . . . . . . . . . 34

Huhdankoski, O. . . . . . . . . 76

Huitema, R. . . . . . . . . . . . 4

Humphreys, G.W. . . . . . . . . 3, 11

Hunt, R. . . . . . . . . . . . . 31

Hunter, J.V. . . . . . . . . . 18, 57

Hunter, M.D. . . . . . . . . . . . . . 12

Hurks, P. . . . . . . 11, 27, 43

Hutchinson, A.J. . . . . . . . . 61

Hutchison, C.W... . . . . . . . 43

Iansek, R. . . . . . . . . . . . 65

Ietswaart, M. . . . . . . . . . . 24

Inder, I.E. . . . . . . . . . . . . 31 
Inder, TE. . . . . . . . . . . . . . . 31

Ingram, L. . . . . . . . . . . . . 12

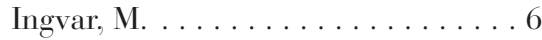

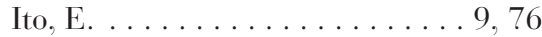

Jacobs, R. . . . . . . . . . 14, 15

Jaffray, M. . . . . . . . . . . . . . 81

Jansari, A.S. . . . . . . . . 10, 26

Jansen, N. . . . . . . . . . . . . . . . 39

Jarosz, J. . . . . . . . . . . . . . . 17

Jayathilake, K. . . . . . . . 37, 70

Jean, L. . . . . . . . . . . . . . . . . 77

Jefferis, S. . . . . . . . . . . . 32

Jenkins, B. . . . . . . . . . . . 72

Jeste, D.V. . . . . . . . . . . . 79

Jodar, M. . . . . . . . . . . . . . 35

Jodzio, K. . . . . . . . . . . . 7, 23

Joe, W. . . . . . . . . . . . . . . 79

Johnson, N. . . . . . . . . . . . . . . . 34

Jolles, J. . . . . . . . . 11, 27, 43, 76

Jones, A. . . . . . . . . . . . . . . . 43

Jones, T.J. . . . . . . . . . . . . . . . 39

Joschko, M.J. . . . . . . . . . . . . . . 81

Joy, P. . . . . . . . . . . . 27

Julien, C. . . . . . . . . . . . . 47

Juncadella, M. . . . . . . . . . . . . 62

Juncadella, M. . . . . . . . . . . . . 58

Junque, C. . . . . . . . 7, 28, 57, 58

Jurado, M. . . . . . . . . . . . . 2

Kajaste, S. . . . . . . . . . . . 40

Kalff, A. . . . . . . . . . . 11

Kalff, A.C. . . . . . . . . . . . 27

Kalmar, J. . . . . . . . . . . . . . . . . 51

Kambanarou, M. . . . . . . . . . . . 42

Kamps, W.A. . . . . . . . . . . 39

Kaneda, Y............. . 37, 70

Kappelle, J.L. . . . . . . . . . . . . . 16

Kappelle, L. . . . . . . . . . . . . . . 38

Kappelle, L.J. . . . . . . . . . . . . 24, 39

Kapur, N. . . . . . . . . . . . . 25, 26

Karaivazoglou, K. . . . . . . . . . . . 39

Karasawa, K. . . . . . . . . . . .9

Karatza, C. . . . . . . . . . . . . . . 39

Karmiloff-Smith, A. . . . . . . . . . . 46

Katati, M.J. . . . . . . . . . . . . . . . 65

Katati, M.J. . . . . . . . . . . . . . 66

Kate, B. . . . . . . . . . . . 62

Kathirgamanathan, U. . . . . . . . . 11

Kato, K. . . . . . . . . . 5, 36

Kaufmann, J.M. . . . . . . . . . . . . 20
Kaufmann, P.. . . . . . . . . . . . . . 32

Kean, M. . . . . . . . . . . . . . 31

Keil, A. ...............20

Keiski, M. . . . . . . . . . . 35

Kelly, T. . . . . . . . . . . . . 72

Kemper, B.E. . . . . . . . . . . 1

Kenardy, J. . . . . . . . . . . 14

Kerber, K. . . . . . . . . . . . 70

Kertesz, A. . . . . . . . . . . . . 48

Kessels, R.C. . . . . . . . . . . . . . . 38

Kessels, R.P.. . . . . 12, 16, 24, 39, 61

Kettle, J.W. . . . . . . . . . . . . . 19

Keuper, O.............. 16

Khoo, S.K. . . . . . . . . . 38

Kidd, N.R. . . . . . . . . . . . . 58

Kiekens, C. . . . . . . . . . . . 58

Kilpatrick, C.. . . . . . . . . . . 19

Kim, H. . . . . . . . . . . . . 60

King, N. . . . . . . . . . . . . . . 72

Kingma, A. . . . . . . . . . . . 39

Kinsella, G. . . . . . . . . . . . 47

Kinsella, G.J. . . . . . . . . . . . . . 75

Kivilahti, J. . . . . . . . . . . . . .51

Klaassen, E.B. . . . . . . . . . . . . . 53

Klein, N. . . . . . . . . . . . . . 32

Knoch, D. . . . . . . . . . . . . 19

Koller, W.C. . . . . . . . . . . . . 64

Kopelman, M. . . . . . . . . . 80

Kopelman, M.D. . . . . . . . . . . 26

Korkman, M. . . . . . . . . . . . 30

Kosten, T.R.. . . . . . . . . . . . . . 13

Koutroumanidis, M. . . . . . . . . 17

Krabbendam, L. . . . . . . . . . . 43

Krum, Н. . . . . . . . . . . . . 40

Kulisevsky, J. . . . . . . . . . . . . 61

Kumada, T. . . . . . . . . . . . . 14

Kumar, A.M. . . . . . . . . . . . . . . 42

Kumar, М. . . . . . . . . . . . . . . . . 42

Kurachi, M. . . . . . . . . . . . 36

Kylmala, M............. 76

Laatu, S. . . . . . . . . . 9 9,51

Laforce, R.. . . . . . . . . . . . 35

LaFrance, M. . . . . . . . . . . . . . 64

Lah, S.S. . . . . . . . . 17

Lamb, A. . . . . . . . . . . . . 81

Lamoureux, J. . . . . . . . . . . . 71

Landro, N.I.. . . . . . . . . . . . . 34

Landry, S. . . . . . . . . . . . 45

Landry, S.H. . . . . . . . . . . 73, 74

Lange, R.............. . 81
Langenecker, S.A. . . . . . . . . . 70

LaPointe, L.L. . . . . . . . . . . . . 1

Lauterbach, M. . . . . . . . . . . 9

Lauterbach, M.H. . . . . . . . . . 22

Lavigne, G. . . . . . . . . . . . 71

Law, C. W. . . . . . . . . . . . 36

Lawlor, B.A. . . . . . . . . . . 62

Lawson, C. . . . . . . . . . . . 24

Lazure, P. . . . . . . . . . . . . . 71

Lee, D.B. . . . . . . . . . . . . . . 42

Lee, T. . . . . . . . . . . . . . 17

Leidig, L.M. . . . . . . . . . . . . . 64

Lejbak, L.K. . . . . . . . . 3, 4, 75

Leroux, C. . . . . . . . . . . . 71

Leventer, R. . . . . . . . . . . 15

Levin, H.S. . . . . . . . . 18, 57

Levine, B. . . . . . . . . . . . 46

Liang, G. . . . . . . . . . . . . . 34

Liddiard, H. . . . . . . . . . . 70

Lincoln, N. . . . . . . . . . . . . 59

Lincoln, N.B. . . . . . . . . . . . . . .56

Loetscher, T. . . . . . . . . . . . 4

Lojek, E. . . . . . . . . . . . . 42, 77

Lokken, K. . . . . . . . . . . . 14

Lopez Jimenez, A. . . . . . . . . . . . 37

Lopez-Gongora, M. . . . . . . . . . . 61

Lopez-Jimenez, A. . . . . . . 41, 65, 66

Lopez-Lorenzo, S. . . . . . . . . . 36

Loureiro, C. . . . . . . . . . . . 9

Love, T.E. . . . . . . . . . . . . 21

Lowe, R. . . . . . . . . . . . . . . 32

Lukatela, K. . . . . . . . . 65

Lund, A. . . . . . . . . . . . 34

Mabbott, D. . . . . . . . . . . . 30

Mabry, J.L. . . . . . . . . . . . . . . 29

MacKay, A. . . . . . . . . . . 8

Mackinlay, R.J. . . . . . . . . . . . 46

Macleod, A.C. . . . . . . . . . . 14

Majerus, S. . . . . . . 20, 44, 63

Mak-Fan, K. . . . . . . . . . . 68

Malec, J.F. . . . . . . . . . . . . . 32, 55

Malefaki, S. . . . . . . . . . 39, 42

Maloney, L.M. . . . . . . . . . . . . 80

Mamolo, C.M. . . . . . . . . . . 52, 53

Man, D. . . . . . . . . . . . 8

Manly, T. . . . . . . . 46, 66

Manning, L............... 9

Manning, P.B. . . . . . . . . . 29

Manning, V..............41

Manzoni, T. . . . . . . . . . . 4 
Marchetta, N............. 43

Marcus, S. . . . . . . . . . . . 70

Marin, J. . . . . . . . . . . . . . 36

Marin, J.J. . . . . . . . . . . . . 36

Marinov, P. . . . . . . . . . . . 41

Markowitsch, H.J. . . . . . . . . . . 26

Marsden, K. . . . . . . . . . . . . . . . 33

Marsden, P. . . . . . . . . 17, 26

Marshall, E.............. 41

Marson, D.C. . . . . . . . . . . . . . 60

Martin, D. . . . . . . . . . . . 81

Martin, E. . . . . . . . . . . . . . 41

Martin-Linares, J. . . . . . . . . . . . 65

Martin-Linares, J. . . . . . . . . . . . 66

Martins, I.P. . . . . . . . . . . . . . 22

Maruyama, T. . . . . . . . . . 14

Masterson, J. . . . . . . . . . . . 80

Mataro, M. . . . . . . . . . . . . . 57

Mathias, J.L. . . . . . . . . . . . 61

Matsui, M. . . . . . . . . . 23, 36

Maunu, M. . . . . . . . . . . . . . . 40

Mayes, A.R. . . . . . . . . . . . 8

Mazer, B.L. . . . . . . . . . . . . . 71

McArthur, I. . . . . . . . . . . . 27

McCarthy, R. . . . . . . . . . . 10

McDonald, S. . . . . . 14, 16, 78

McGregor, K. . . . . . . . . . 21

McHugh, T. . . . . . . . . . . 35

McKinlay, L. . . . . . . . . . . . 14

McLoughlin, D.M. . . . . . . . . . . 35

McMillan, T. . . . . . . . 48,55

McMillan, T.M. . . . . . . . . . . 49

McMonagle, P. . . . . . . . . . . . 48

McNaughton, N. . . . . . . . . 1

Meersmans, M. . . . . 37, 41, 65, 66

Meijer, W. . ........... 76

Meijs, C. . . . . . . . . . . 11

Melissa, B. . . . . . . . . . . 65

Melo, B. . . . . . . . . . 67, 68

Meltzer, H. . . . . . . . . 37, 70

Mendonca, A. . . . . . . . 6, 10

Meneses, R.F. . . . . . . . . . . 13, 50

Mercader, J. . . . . . . . . . . . . 28

Merino, V. . . . . . . . . . . . . 36

Merten, T. . . . . . . . . . . . . 69

Messinis, L. . . . . . . . . . . . . 39, 42

Meyer, A. . . . . . . . . . . 33

Meyers, J.E. . . . . . . . . . . . 2

Michiels, K. . . . . . . . . . . 58

Mickley, N. . . . . . . . . . 11

Mijch, A. . . . . . . . . . 43

Milders, M. . . . . . . . . 12, 24
Miller, C. . . . . . . . . . . . . . . . . 29

Miller, L. . . . . . . . . . . . . . . . 17

Minguez-Castellanos, A. . . . . . 65, 66

Minich, N. . . . . . . . . . . . . 15

Miranda, R. . . . . . . . . . . . . 62

Mitchell, D.J. . . . . . . . . . . . 1

Mitchell, S.A. . . . . . . . . . 38

Mizuta, Н. . . . . . . . . . . . ?

Moes, E. . . . . . . . . . . . . 52

Moessner, A.M. . . . . . . . 32, 55

Moffat, C. . . . . . . . . . . . . 27

Mogg, A. . . . . . . . . . . . . . 35

Molfese, D. . . . . . . . . . . . . 44

Molfese, V. . . . . . . . . . . . 44

Molina, R. . . . . . . . . . . . . 42

Moloney, P. ............. . 7

Monsch, A.U. . . . . . . . . . . . 9

Montagne, B. . . . . . . . . . . . . 24

Montaldi, D. . . . . . . . . . 8

Moore, A.B. . . . . . . . . . . . 21

Moore, N.B. . . . . . . . . . . . . . 51

Moral, M.. . . . . . . . . . . . 62

Moratti, S. . . . . . . . . . . 20

Mordhorst, M. . . . . . . . . . . 70

Mori, E. . . . . . . . . . . . . 10

Morin, A. . . . . . . . . . . 71

Morris, J. . . . . . . . . . . . . . 3

Morris, L. . . . . . . . . . . . . . . 59

Morris, R. . . . . . . . 17, 54

Morrison, T. . . . . . . . . . . . 59

Morriss, E. . . . . . . . . . . 14

Morse, S. . . . . . . . . . . . . 15

Mortensen, E.L. . . . . . . . . . . . . 62

Moseley, A. . . . . . . . . . . 78

Motomura, N. . . . . . . . . . 7

Mouratidis, M. . . . . . . . . . . 13

Mueller, S.C. . . . . . . . . . . . . 4

Mulder, T. . . . . . . . . . . . 4

Munday, R. . . . . . . . . . . . 49

Mungas, D. . . . . . . . . . . . 61

Muragaki, Y. . . . . . . . . . . 14

Murdoch, B.............. 58

Murray, J. . . . . . . . . . . . . . 72

Myran, D. . . . . . . . . . . 33

Naalt, van der, J. . . . . . . . . . . . 12

Nagahara, N. . . . . . . . . 9, 76

Narberhaus, A. . . . . . . . 7, 28

Narvaez, J.M. . . . . . . . . . . . . . 79

Neary, D. . . . . . . . . . . . . . 47

Neggers, S.F. . . . . . . . . . 16
Neo Suat Hong, P. . . . . . . . . . . . 1

Newcombe, K. . . . . . . . . . . 40

Newhoff, M............... . 21

Newson, M. . . . . . . . . . . . 64

Ngo, L. . . . . . . . . . . 67, 68

Nielsen, K. . . . . . . . . . . . 4 43, 44

Nielson, K.A. . . . . . . . . . . . 70

Niki, C. . . . . . . . . . . . . 14

Nishimura, R. . . . . . . . . . 5

Nishiyama, S. . . . . . . . . . . 23

Nunez-Nunez, V. . . . . . . . . . . . . . 12

Nuss, K... . . . . . . . . . . . . 28

Nys, G.M. . . . . . . . . . . . . . 24

O'Connell, H. . . . . . . . . . 62

O'Keeffe, F. . . . . . . . 54, 55, 58

O'Keeffe, F.M. . . . . . . . . . . . . .

O’Mellan, S. . . . . . . . . . 42

O'Neill, S.M. . . . . . . . . . . 38

O'Toole, K.M. . . . . . . . . . . . 29

Obler, L.K. . . . . . . . . . . . . . 23

Obonsawin, M.C. . . . . . . . . . . 32

Ogden, J. . . . . . . . . . . . . . 50

Ohan, J. . . . . . . . . . . . . . 15

Okuda, J. . . . . . . . . . . 67

Ong, B. . . . . . . . . . 47, 75

Oosterman, J. . . . . . . . . . 75

Orban, G. . . . . . . . . . . 67

Orlebeke, J.F. . . . . . . . . . . . 75

Orozco-Gimenez, C.. . . . . 4 41, 65, 66

Ortega Lozano, S. . . . . . . . . . . . 37

Ortega Moreno, A. . . . . . . . . . 66

Ortega-Moreno, A...........665

ОТА, Н............. 5

Owen, A. . . . . . . . . 48, 49, 72

Ownby, R.L. ............. 42

Ownsworth, T........ 54,55

Pachana, N.A. . . . . . . . . . 14, 38

Paggi, A. . . . . . . . . . . . 4

Pagulayan, K.F. . . . . . . . . . . . 56

Pahlson, A. . . . . . . . . . . . 38

Paolo, A.M. . . . . . . . . . . . . 64

Park, N.W. . . . . . . . . . 52, 53

Park, S. . . . . . . . . . . . 37

Parker, R.S. . . . . . . . . . . 41

Parkinson, R.B. . . . . . . . . . 21

Parks, R.W. . . . . . . . . . . . 12

Pascual-Sedano, B. . . . . . . . . 61

Passant, U. . . . . . . . . . . . . 17

Patrick, P.D. . . . . . . . . . . . 29 
Patterson, K.................. 80

Paulsen, J.S. . . . . . . . . . . . . . 2

Pavao Martins, I. . . . . . . . . . . . . . 9

Pavlovic, M.. . . . . . . . . . . . . 31

Peach, R.K. . . . . . . . . . . 34

Peck, A.W. . . . . . . . . . . . 38

Peck, E.A. . . . . . . . . . . . . . . 38

Peck, K.K. . . . . . . . . . . . . 21

Peck, L.W. . . . . . . . . . . . . 38

Pedersen, A.D. . . . . . . . . . . . 76

Pena, J. . . . . . . . . . . . . . . 62

Pender, N. . . . . . . . . . . . . . . . 48

Penkman, L. . . . . . . . . . . . . . 30, 79

Perdices, M. . . . . . . . . . . . . 78

Perez Garcia, M. . . . . . . . . . . . . 20

Perez, M. . . . . . . . . . . . . . . . . 37

Perez-Garcia, M. . . . . . . . 41, 65, 66

Perry, J. . . . . . . . . . . . . . . 8

Peters, F. . . . . . . . . . . . . 63

Petersson. K. . . . . . . . . . . . . 6. 10

Petkova, P............... 41

Pickard, J. . . . . . . . . . . . . . . . 48

Pluck. G. . . . . . . . . . . . . . 35

Poca, A. . . . . . . . . . . . . . . 57

Polo-Kantola, P.. . . . . . . . . . . . 76

Poncelet, M. . . . . . . . . . . . . . . . . 44

Portin, R... . . . . . . . . . . 76

Postema, K. . . . . . . . . . . . 4

Postma, A. . . . . . . . . 16, 61

Poutiainen, E.T. . . . . . . . . . . . . 40

Powell, M.R. . . . . . . . . . . . 71

Power, T. . . . . . . . . . . . . . . 15

Price, J.R. . . . . . . . . . . . . . 81

Pueyo, R. . . . . . . . . . . . . . 57

Purdy, M. . . . . . . . . . . . . . 21

Putnam, S.H. . . . . . . . . . . . 37

Quinn, S.................... 35

Radloff, S. . . . . . . . . . . . . 57

Rado, T.. . . . . . . . . . . . . . . . 41

Rafal, B. . . . . . . . . . . . . 54

Rafal, R. . . . . . . . . . . . . 54

Rahman, R. . . . . . . . . . . . 5

Ramos Font, C. . . . . . . . . . . . 37

Ramsden, C. . . . . . . . . . . . 47

Rao, H.Y. . . . . . . . . . . . . . . 19

Rapport, L.J. . . . . . . . . . . . . 70

Raskind, W. . . . . . . . . . . 43

Raycheva, M. . . . . . . . . . . . 41

Raymer, A.M.. . . . . . . . . . . . . . 22
Recknor, E.C. . .............. 20

Reed. L. . . . . . . . . . . . . . . . . . . 17

Reed, L.J. . . . . . . . . . . . . 26, 41

Regard, M. . . . . . . . . . 4, 11

Reimer, M. . . . . . . . . . . 48, 49

Reis, A.I. . . . . . . . . . . . 6, 6, 10

Rene, R... . . . . . . . . . . . . 62

Ribeiro, J.P. . . . . . . . . . . . . . 13

Ribera, G. . . . . . . . . . . . . 35

Rico-Blanco, B. . . . . . . . . . . . . 12

Rinne, R. . . . . . . . . . . . . . 51

Ris. M. . . . . . . . . . . . . . . 40

Risberg, J. . . . . . . . . . . . . 17

Ritter, E. . . . . . . . . . . . . . 9

Rivlin, E. . . . . . . . . . . . 27

Roane, D. . . . . . . . . . . . 65

Roberts, N. . . . . . . . . . 8

Robertson, I. . . . . . 7, 24, 25, 46, 55

Robinson, S.R. . . . . . . . 43, 62

Robison, M.A. . . . . . . . . . . 27

Rodriguez Fernandez, A. . . . . . . . 37

Rodriguez, A. . . . . . . . . . . 22, 58

Roig Rovira, T. . . . . . . . . . . . . 78

Roine, R.O. . . . . . . . . . . . . . . 40

Roness. A. . . . . . . . . . . . . . . . 34

Rooijakkers, L. . . . . . . . . . . 43

Rosenfeld, J. . . . . . . . . . . . . . . . 15

Ross, S.P. . . . . . . . . . . . 4

Ross, S.R. . . . . . . . . . 37

Round, A. . . . . . . . . . . . . . 72

Rourke, B.P.. . . . . . . . . . . . . 73

Roy, E.A. . . . . . . . . . . 52,53

Rubio, F. . . . . . . . . . . . 58

Rund, B.R... . . . . . . . . . . . 34

Rushworth. M.F. . . . . . . . . . . 46

Rusin. J. . . . . . . . . . . . . . . . 28

Russell, A. . . . . . . . . . . . . . 70

Ryan, J.D. . . . . . . . . . . . . . 59

Sahuquillo, J. . . . . . . . . . . . 57

Saint-Cyr. J.. . . . . . . . . . . . . . 13

Salmon, E... . . . . . . . . . . 63

Samson, D. . . . . . . . . . . . . . 11

Sanchez-Carrion Abascal, R. . . . . . 78

Sandoval, L. . . . . . . . . . . . . 14

Sanusi, N. . . . . . . . . . . . . . 50

Sanz de la Torre, J. . . . . . . . . . . . 36

Sanz, J.C. . . . . . . . . . . . . . . . 36

Saunders, C. . . . . . . . . . . . . 16

Scarcia, M.J. . . . . . . . . . . . . 73

Scherder, E. . . . . . . . . . . . . 75
Scherder. E.J.. . . . . . . . . . . . 63. 75

Schluchter, M. . . . . . . . . . . . . 32

Schmidt, B.T. . . . . . . . . . . 23

Schnider, A. . . . . . . . . . . . . . 17

Scholvinck, M. . . . . . . . . 67

Schwartz, R. . . . . . . . . . 21

Schweiger, A. . . . . . . . . . . . 71

Schweinberger, S.R. . . . . . . 6, 6, 20

Scott-Lane, L. . . . . . . . . . . . . . . 79

Segarra, D. . . . . . . . . . . . 7

Seiler, C.B... . . . . . . . . . . . . 1

Senior, G.. . . . . . . . . . . . . . . . 81

Senn. T.E. . . . . . . . . . . . . 28

Sergeant, J.A.. . . . . . . . . . . 75

Serra-Grabulosa, J. . . . . . . . . . . 2

Shapiro, L.P. . . . . . . . . . . . 21

Shaw, M. . . . . . . . . . . . . 75

Shawaryn, M. . . . . . . . . . . . 51

Shen, I. . . . . . . . . . . . . . . . . . 29

Sherlock, R.L. . . . . . . . . . . . . 31

Sheth, A. . . . . . . . . . . . . 18

Shin. M. . . . . . . . . . . . 60

Shore, D.L. . . . . . . . . . . . . . . 35

Shores, E. . . . . . . . . . . . . 27

Shores, E.A. . . . . . . . . . . . . 69

Shum, D. . . . . . . . . . . . . . . 66

Shuttleworth-Edwards, A. . . . . . . 57

Silfvast, T. . . . . . . . . . 40

Simard, M. . . . . . . . . . . 33, 77

Simmons, Z. . . . . . . . . . . . . 52

Simon, E.S. . . . . . . . . . . . . 71

Simons, J.S. . . . . . . . . . . . . . 81

Simpson, S.A. . . . . . . . . . . 81

Singletary, F. . . . . . . . . . . . . 22

Sitzer, D.I. . . . . . . . . . . . . . . . . 79

Skelton, R.W.. . . . . . . . . 4, 81

Skibinski, G. . . . . . . . . . . . . . . 47

Skilbeck, C.... . . . . . . . . . 33

Skilbeck, C.E. . . . . . . . . . . . . . 58

Slaats-Willems, D. . . . . . . . . . 27

Slaats-Willemse, D. . . . . . . . . . 11

Slatyer, M. . . . . . . . . . . . . . 33, 58

Smart, L.A. . . . . . . . . . . . . . 70

Smigielski, J.S. . . . . . . . . . . 71

Smit, M.C. . . . . . . . . . . . . 53

Smith-Chant, B. . . . . . . . . . 45

Smits, A. . . . . . . . . . . . 38

Snowden, J. . . . . . . . 47

Sofuoglu, M. . . . . . . . . . 13

Sopena, S. . . . . . . . . 70

Spaan, P.E. . . . . . . . . . 74

Spaeth, J.P. . . . . . . . . . . . 29 
Spence, S.A. . . . . . . . . . 12

Spikman, J.M. . . . . . . . . . . . 12

Spooner, D.M. . . . . . . . . . . 38, 39

Stancin, T. . . . . . . . . . . 15

Stanhope, N. . . . . . . . . . . . 26

Stefanatos, G. . . . . . . . . . . . 79

Stevenson, R. . . . . . . . . . . 24

Stierwalt, J.A. . . . . . . . . . . . 1

Stigsdotter Neely, A. . . . . . . . . 78

Stokholm, J. . . . . . . . . . . . 63

Stolwyk, R.J. . . . . . . . . . . . . 65

Stone, L. . . . . . . . . . . . . . . 52

Stopford, C. . . . . . . . . . . 47

Stordal, K.I.. . . . . . . . . . . . . 34

Storey, E. . . . . . . . . . . . . . 40

Stringer, K. . . . . . . . . . . . 69

Strother, D. . . . . . . . . . . . . 30

Subira, S. . . . . . . . . . . 35

Sumpter, R. . . . . . . . . . . . . . 55

Sundet, K.S. . . . . . . . . . . . . 34

SUZUKI, K........... 5

Suzuki, K. . . . . . . . . . . . 10

Suzuki, M. . . . . . . . . . . . 10

Swaab, D.F. . . . . . . . . . . 75

Tagliati, M. . . . . . . . . . . . 65

Takeuchi, A. ............ 36

Tallus, M. . . . . . . . . . 76

Tam, S. . . . . . . . . . 8

Tate, D.F. . . . . . . . . . . . 18

Tate, R.L. . . . . . . . . . . 78

Taub, A. . . . . . . . . . . . . . 69

Taylor, G. . . . . . . . . . . . . 14

Taylor, G.H. . . . . . . . . . . . . . . 15

Taylor, H. . . . . . . . 28 28, 73, 74

Taylor, H.G.. . . . . . . . . . . 30, 32

Tellegen, P.J. . . . . . . . . . . . . . 39

Temkin, N.R. . . . . . . . . . . 56

Tersiysky, R. . .......... 41

Theocharis, G. . . . . . . . . . . 39

Thomas, A. . . . . . . . . . . . . 58

Thomas, M. . . . . . . . 33, 58

Thomas, S.A. . . . . . . . . . . . 59

Thomason, K.E. . . . . . . . . . . 50

Thompson, A.L. . . . . . . . . . . 53

Thompson, D. . . . . . . . . . . 31

Thompson, J. . . . . . . . . . 4 7

Tiainen, M. . . . . . . . . . . 40

Till, C. . . . . . . . . . . . . . 68

Tippett, L. . . . . . . . . . . . 50

Togher, L. . . . . . . . . . . . . . . . 78
Tomaszewski Farias, S. . . . . . . . 61

Triggs, T.J.............. 65

Troster, A.I. . . . . . . . . . . . . . 64

Tsai, C. . . . . . . . . . . . . . 34

Tsakona, I.. . . . . . . . . . . 42

Turgeon, M. . . . . . . . . . . . 37

Turner, M. . . . . . . . . . . . . . 72

Twamley, E.W. . . . . . . . . . . . 79

Tyler, J. . . . . . . . . . . . 32

Ungerleider, R... . . . . . . . . . 29

Urrila, A. . . . . . . . . . . . 76

Van Asselen, M. . . . . . . . . . . . . 16

Van Boxtel, M. . . . . . . . . . . 76

Van den Berg, E. . . . . . . . . . . . 38

Van den Kommer, T.N. . . . . . . . . 63

Van der Beek- Boter, J.W.. . . . . . . . 39

Van der Knokke, S. . . . . . . . . . . 75

Van der Linden, M. . . . . . . . . 8, 20

Van Gerven, P. . . . . . . . . . . 76

Van Paasschen, J. . . . . . . . . . . 75

Van Walsem, R.M. . . . . . . . . . . 63

Van Zandvoort, M. . . . . . . . . . 22

Van Zandvoort, M.J. . . . . . . . 12, 24

Vancoillie, P. . . . . . . . . . . 55

Vanier, M. . . . . . . . . . . . . 71

Vanschoenbeek, K. . . . . . . . . . 55

Vargas, M. . . . . . . . . . . . . 36

Vargas, M.L. . . . . . . . . . . 36

Vassileva, J. . . . . . . . . . . . . . 41

Veerman, A.J. . . . . . . . . . . . . . 39

Velinov, V. . . . . . . . . . . 41

Vendrell, P. ............. 2

Vera-de-la-Puente, E. . . . . . . . . 12

Verdejo, A.J. . . . . . . . . . . . 20

Verdejo-Garcia, A. . . . . . . . . . 41

Verhoeff, P. . . . . . . . . . . 33

Vesey, P.A. . . . . . . . . . . . . 56

Vidal, C. . . . . . . . . . . . . 78

Vilar, R. . . . . . . . . . 65

VILAR, R. . . . . . . . . . 37

Vilar, R........... 41, 66

Villa-Moreira, S. . . . . . . . . . . 12

Villani, F. . . . . . . . . . . . . . . 50

Vinas, M.J. . . . . . . . . . . . . 36

Vogel, A. ...........62,63

Vrbancic, M. .......... 3, 4

Wade, S. ............... 15

Waldemar, G.

62,63
Waldrop, D. . . . . . . . . . 42

Walker, N.B. . . . . . . . . . . 10

Wall, S.E. ............ 72

Walsh, J.B. . . . . . . . . . . . 62

Wang, H. . . . . . . . . . . . . 31

Wang, Y. . . . . . . 4 45, 57, 69

Wang, Z.J. . . . . . . . . . . . . 18

Wanigaratne, S. .......... 41

Ward, T. . . . . . . . . . . . 26

Wassenberg, R. . . . . . . . . . 27

Watanabe, H............... 9

Weaver, L.K. . . . . . . . . . . . 18

Weintraub, S............. 34

West, C. . . . . . . . . . 27

Wester, A. . . . . . . . . . . . 12

Wester, A.J. . . . . . . . . . . 16

Whalley, L.J. . . . . . . . . . . 74

Wheatley, A.C............ 3

White, D.............. 8

White, D.A. . . . . . . . 59

White, K. . . . . . . . . . . 21

Whittier, N. . . . . . . . . . 51

Whittier, N.C. . . . . . . . . . 51

Wiarda, M.G. . . . . . . . . . 63, 71

Wiebe, D............... 18

Wiebe, S. . . .......... 45

Wierenga, C. . . . . . . . . . . 21

Wilde, E. . . . . . . . . . 18

Wilkie, F.L. . . . . . . . . . 42

Williams, H.. . . . . . . . . 56, 72

Willmes, K. . . . . . . . . . . 22

Wilson, B.A...... 48, 54, 56, 70

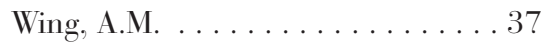

Withall, A............. 34

Witol, A. . . . . . . . . . 30

Woldt, K. . . . . . . . . 32, 46

Woll, B. ................ 22

Woodruff, P.W............... 12

Woodward, L.J. . . . . . . . . 30, 31

Worthington, E. . . . . . . . . . 32

Wright, E. . . . . . . . . . . 43

Wright, H.H. . . . . . . . . . . . 21

Wright, I. . . . . . . . . . . . 50

Wright, J.P. . . . . . . . . . 40

Wright, M. . . . . . . . . . . 28

Wright, M.J.. . . . . . . . . . . 39

Yamadori, A. . . . . . . . . . . . . 5

Yang, Y. . . . . . . . . . . . . 34

Yates, P.J. . . . . . . . . . . . . 72

Ye, B.B. . . . . . . . . . . . . . 19

Yeates, G.N. . . . . . . . . . . . 33 
Yeates, K. . . . . . . . . . . 14, 28

Yeates, K.O. . . . . . . . . . . . . 15, 73

Yelland, G.W.. . . . . . . . . . 43, 62

Yodkovik, N. . . . . . . . . . . 70

Yoshizaki, K. . . . . . . . . . . 5

Young, E.A. . . . . . . . . . . . . 70

Yuuki, H. . . . . . . . . . . . 36

Zamrini, E.Y.. . . . . . . . . . . 60

Zheng, W. ............... 42

Zoratti, S. . . . . . . . . . . . 35

Zuberi, S. . . . . . . . . . 27

Zubieta, J. . . . . . . . . . . . 70 